\title{
Impacto de las redes sociales en el sector del enoturismo del Priorat.
}

\section{Impact of social networks in the wine tourism sector of Priorat}

Joan Quero Guerrero. ${ }^{1}$, Julio Cesar Caicedo Aldaz. ${ }^{2}$, Lenin Landivar Cedeño Alcivar. ${ }^{3}$ \& Cristian Roberto Zúñiga Chamorro. ${ }^{4}$

\section{DOI: https://doi.org/10.33262/visionariodigital.v3i3.647}

\begin{abstract}
ABSTRAC
The emergence of wine tourism has meant for the Catalan wine sector enter a new stage where you have to understand what is the expected product, how the customer expects it and where companies have to go to look for customers. It is a segment on the rise, which needs a constant and often discreet work. How can social networks position a company and differentiate it from the rest? Are the Priorat companies doing it? To answer these questions, we will proceed with an exploratory analysis where we will know how communication channels use the wine tourism companies of this region with renown in the wine world but with figures still low in wine tourism throughout the year compared to other areas of wine. Catalan and Spanish wines such as Alt Empordà or La Rioja. We will open this door to find out what is on the other side.
\end{abstract}

Key words: Wine sector, wine sector in Catalonia, vertebrate of oenological routes, Priorat companies, wine tourism sector.

\section{Resumen.}

La eclosión del enoturismo ha supuesto para el sector vitivinícola catalán entrar en una nueva etapa dónde se tiene que comprender cuál es el producto que se espera, cómo lo espera el cliente y dónde tienen que ir las empresas a buscar a los clientes. Es un segmento en alza, que necesita de un trabajo constante y, a menudo, discreto. ¿Cómo pueden las redes sociales posicionar a

1 Universidad de Barcelona, Facultad de Ciencia Administrativas y Económicas, Barcelona- España, guerreroj@ub.edu.ec

${ }^{2}$ Universidad Técnica Luis Vargas Torres, Sede Santo Domingo, Facultad de Ciencias Administrativas y Económicas, Santo Domingo - Ecuador, juliocesarcaicedo@ hotmail.com

${ }^{3}$ Universidad Técnica Luis Vargas Torres, Sede Santo Domingo, Facultad de Ciencias Administrativas y Económicas, Santo Domingo - Ecuador, leninced62@hotmail.com

${ }^{4}$ Universidad Técnica Luis Vargas Torres, Sede Santo Domingo, Facultad de Ciencias Administrativas y Económicas, Santo Domingo - Ecuador, cristian_zuniga82@hotmail.com 
una empresa y diferenciarla del resto? ¿Lo están haciendo las empresas del Priorat? Uno de los ejes dónde aún se encuentran lejos de Burdeos o la Toscana es con el nivel de conexión con los clientes. En este trabajo analizaremos esta conexión des del punto de vista de las redes sociales, un paradigma aún por trabajar y que tiene dos hándicaps de primera mano. Así mismo Ylera (2007) define enoturismo como un fenómeno en el que los mundos del vino y turismo. Se funden para ofrecer una combinación de sus respectivas culturas, magias y atractivos. Otros autores mantienen la misma idea a la hora de definir el enoturismo como Imagen, ocio, cultura, salud y muchas cosas que acompañan al vino y que en cierto Modo todavía en España no se sabe aprovechar (Del Rey y Latre, 2007).

Palabras clave: Sector vitivinícola, sector del vino en Cataluña, vertebración de las rutas enológicas, las empresas del Priorat, sector enoturistico.

\section{Introducción}

El sector del vino en Cataluña se encuentra en auge, se exporta más que nunca y sus marcas son conocidas mundialmente. La pregunta es: ¿La masa turística que le acompaña también está aprovechada? En este trabajo se analiza las claves por lo que los turistas se lanzan a realizar enoturismo y qué estrategias usan las bodegas catalanas en las redes sociales, más concretamente en el Priorat. Administraciones y entes locales han promovido este sector que ha dinamizado económicamente comarcas catalanas que se habían despoblado históricamente, pero aun así todavía están lejos de alcanzar los números del enoturismo que tienen zonas, igual de conocidas que aquí, como Francia e Italia.

Uno de los ejes dónde aún se encuentran lejos de Burdeos o la Toscana es con el nivel de conexión con los clientes. En este trabajo se analiza esta conexión desde el punto de vista de las redes sociales, un paradigma aún por trabajar y que tiene dos hándicaps de primera mano. Tenemos primero el poco desarrollo de la cobertura en el Priorat, su orografía hace que la máxima velocidad de internet no llegue a todos los puntos del territorio, un punto que se está mejorando, pero sin duda una barrera importante para estar presente en las redes sociales. Otra barrera que podemos encontrar es la desconfianza empresarial hacia las estrategias la sociedad media por la dificultad de medir el retorno económico. También analizamos el impacto del uso de las redes sociales con aquellos clientes actuales y potenciales del enoturismo. Para concretarlo nos centraremos en una zona geográfica particular, el Priorat, muy conocida por sus vinos, pero con mucho potencial aún por explotar en el terreno del enoturismo.

\section{Marco Teórico Referencial}

La definición de enoturismo no se puede entender sin conocer antes los conceptos de marketing de las empresas vitivinícolas y las motivaciones que tienen los viajeros. Una posible aproximación al término podría ser la interpretación que Hall (2000) hace en Wine tourism arround the world: "El turismo del vino es la experiencia de visitar viñedos, conocer bodegas, asistir a festivales y a demostraciones vinícolas en los cuáles la cata del vino y/o la experiencia de los atributos del mismo son el principal motivo para los visitantes". Podríamos relacionar el enoturismo con las zonas geográficas y su reactivación económica 
haciendo un modelo turístico sostenible y ético. El impacto socioeconómico de este tipo de turismo no sólo beneficia a las industrias vitivinícolas, sino que reporta cambios a toda la región. También existen otras formas de abordar el término de enoturismo, encontramos otras acepciones entre autores españoles. Así mismo Ylera (2007) define enoturismo como un fenómeno en el que los mundos del vino y turismo se funden para ofrecer una combinación de sus respectivas culturas, magias y atractivos. Otros autores mantienen la misma idea a la hora de definir el enoturismo como imagen, ocio, cultura, salud y muchas cosas que acompañan al vino y que en cierto modo todavía en España no se sabe aprovechar (Del Rey y Latre, 2007). Esta última afirmación hace reconducir la idea de que esta clase de turismo no sólo beneficia a la bodega, sino que actúa como potente dinamizador de la economía del territorio.

El turismo, pero no ha sido una prioridad en la industria vitivinícola europea hasta hace una década. El impulso se generó gracias a los países del "nuevo mundo" del vino (Getz and Brown, 2006). El planteamiento de la actividad ha sido desigual entre las dos grandes regiones vitivinícolas del mundo: Europa y el Nuevo Mundo. Existen condicionantes que han hecho que el éxito de este proceso se haya desarrollado más rápidamente en los países del nuevo mundo que en Europa. Estos condicionantes pueden ser el gran nivel de turismo, pero también otros aspectos que hace falta sacar a luz para determinar qué es lo que se puede mejorar en el sector enoturistico más tradicional.

\section{El Enoturista}

La industria del vino y su potencial turístico lleva a masas de turistas a identificar sus vacaciones junto a un complejo vitivinícola. Los análisis del mercado realizados hasta hora sugieren que no hay un enoturista "tipo", sino que es la demanda por la que se puede segmentar y clasificar los turistas del vino. Serrano (2011) propone los aspectos siguientes para definir al enoturista:

a) Se desplaza hasta el lugar de producción del vino

b) el desplazamiento implica un cambio en la rutina del turista del vino y una influencia en los factores externos en el momento de realizar su experiencia.

c) se identifica con el cambio de actitud del turista en el momento de asimilar la experiencia

d) el enoturista requiere servicios complementarios para que su experiencia sea placentera y la recuerde, es por eso que el vino no es el único elemento decisorio.

\section{Concepto de región enoturística}

El posicionamiento competitivo de las regiones enoturísticas se ha convertido en un elemento estratégico muy importante para atraer a los turistas. La importancia de la región se centra en el reconocimiento del prestigio de sus vinos, pero también de la historia, la gastronomía y el escenario. Con esto nos preguntamos ¿por qué unas regiones son más visitadas que otras con vinos del mismo nivel de reconocimiento? La respuesta la encontramos en la transformación del entorno, hay regiones centradas en transformar con cuidado los elementos estéticos del 
paisaje para otorgar las mejores experiencias de visita, la imagen vale más que mil palabras para describir una experiencia, incluso en el turismo del vino.

Pero ¿Por qué son importantes las DO? El elemento diferenciador entre regiones vitivinícolas es la denominación de origen que determina una combinación de las características físicas, naturales y culturales que representa un destino. Mitchell y Hall (2002) lo denominan terror turístico y esto es uno de los ejemplos más claros de la importancia creciente del marketing en regiones para atraer nuevos turistas. Cuando se habla de terror turístico los aspectos claves que determinan la atracción turística son la tipicidad y la personalidad. La tipicidad son las características únicas que diferencian al territorio y que le atribuyen una identidad. Esto nos lleva al segundo elemento clave, la personalidad, que ayuda a configurar una demanda turística determinada.

El otro problema al cual se encuentran las bodegas es que hay una infinidad de vinos de calidad que causa mucha confusión en el consumidor. La solución a este problema pasa por la categorización y clasificación de las calidades, de esta forma la DO tendrá como objetivo la diferenciación del producto, así como el de ofrecer un sello de calidad.

\section{Enoturismo en España}

En las últimas décadas, la industria del vino en España se ha internacionalizado de manera significativa. Aun así, la industria mundial evoluciona continuamente y aparecen nuevos y grandes competidores que implementan estrategias de comercialización mucho más orientadas al marketing, la comunicación y la marca.

Los objetivos iniciales que se buscaban con la vertebración de las rutas enológicas se centraron en la coordinación entre las distintas bodegas existentes en un área geográfica. Posteriormente se fueron incorporando otros objetivos a estas rutas enoturísticas, como el concepto técnico de producto turístico, elemento clave en un país caracterizado por la importancia de la actividad turística, el establecimiento de un mecanismo de coordinación entre las diferentes rutas o la creación de un producto de calidad que permitiese aumentar la oferta cultural de España, uniéndola con su tradición vitivinícola. Para reforzar este concepto de calidad se creó, a través de un acuerdo entre el Gobierno de España y determinadas entidades privadas relacionadas con diferentes rutas enoturísticas el Observatorio Turístico Rutas del Vino de España que pretende controlar el desarrollo de este producto turístico para adaptarlo a los estándares de calidad que se pretende conseguir. Asimismo, y junto con este objetivo de control, el Observatorio suministra, a través de diferentes estudios, una interesante información relacionada tanto con la oferta como con la demanda existente en este campo. En los últimos años estamos asistiendo a un cambio en el comportamiento de los turistas que buscan otro tipo de destinos con la finalidad de encontrar nuevas experiencias, y ello está provocando, además de viajes de menor duración, visitas en áreas geográficas hasta ahora alejadas de los tradicionales destinos turísticos que hace se comience a posicionar una nueva tipología de turismo. 
Análisis del sector en Cataluña

Los principales retos a los cuales se enfrentan las bodegas y los destinos enoturísticos de Cataluña para competir con las principales regiones enoturísticas son: incrementar el volumen de turistas extranjeros, aumentar el gasto medio por enoturista y alargar las estancias. Cataluña es una de las regiones europeas con mejores índices de calidad de vino. Es por esto que, aunque los datos son buenos, el sector debe encontrar las palancas para sacar más provecho de un tipo de turismo que atrae cada vez a más turistas. El cliente de turismo enológico en Cataluña es básicamente nacional. El reto está, pues, en ofrecer un producto adecuado a las necesidades de los turistas extranjeros.

El segundo objetivo que se marcan los destinos enoturisticos españoles es incrementar el gasto medio por turista que está muy por debajo del gasto medio en otros destinos europeos. Aunque en 2017 se mejoraron las cifras alcanzando un gasto medio de 156 al día frente a los 144,3 del año anterior, los datos siguen estando lejos de los estándares de Italia y Francia. En este mismo aspecto, según los datos de la Asociación española de ciudades de vino, el enoturismo se sigue comportando como una modalidad turística de escapada, un producto asociado a fines de semana y puentes con una media de 2,65 días. La falta de estas pernoctaciones implica más daño aún a los tres principales pilares del enoturismo: bodegas, restaurantes y alojamientos. Para alargar más estas estancias y lograr estos retos, hay una serie errores habituales en los destinos catalanes que hacen que no se logren las cifras envidiables de otras regiones.

\section{Optimismo de cara al futuro.}

El enoturismo en Cataluña va dando pasos cada vez más conjuntamente con el fin de beneficiar a toda una zona con gran potencial como es el territorio catalán. Iniciativas como el VIVID, el festival de enoturismo de la costa Brava ha elevado la experiencia enoturística hasta el punto de empezarse a comparar a otras regiones como La Rioja o la Vall de Loira con gran experiencia en este campo. Según el informe de la Agencia catalana de turismo del 2016, un $95 \%$ de los enoturistas que han visitado Cataluña tienen la intención de volver. Esto es un auténtico éxito para las bodegas catalanas que ven cómo la marca Cataluña cada vez es más conocida entre los enoturistas europeos.

\section{Marketing del enoturismo}

Apostar por el enoturismo pasa por generar un plan estratégico de marketing que ayude a diferenciarse de la competencia, o lo que es lo mismo, a tener mayores posibilidades de éxito. El marketing en el sector enoturístico es mucho más que el simple packaging o asistencia en ferias, implica interacción continuada, engagement y emoción.

\section{El producto de enoturismo}

El producto de turismo es el elemento esencial del marketing-mix en el agroturismo; todos los elementos "clásicos" se determinan estrictamente porque el producto se distribuye, promociona y se establece el precio. El producto turístico debe satisfacer la diversidad de las necesidades de los consumidores y para que "el productor" obtenga un beneficio ventajoso, debe determinar la naturaleza multidimensional de la demanda y suministrar sólo aquellos bienes que reclaman 
los turistas. El producto de turismo ofrecido por el turismo rural, incluye un conjunto de productos y servicios (manutención, alojamiento, entretenimiento, etc.) explotado dentro de un contexto específico, influido por los factores naturales de una región o de un lugar. La calidad del producto turístico está directamente relacionada con la explotación del patrimonio turístico de la zona, considerando los recursos naturales, la infraestructura general y sobre todo la calidad de los recursos humanos implicados en la actividad turística.

\section{Entorno tecnológico}

Toda decisión para comprar un producto en internet se basa en la relación emocional-racional. El éxito de adquirir los productos turísticos rurales se basa sobre todo en la imagen y experiencia de las personas que vayan a efectuar la compra y también de la forma en que ofrece una satisfacción a cada uno de los compradores. La promoción de productos de enoturismo en la red recae algunas veces por la misma bodega y otras por intermediarios. De este modo toma importancia el intermediario ya que la promoción del producto recae directamente en la imagen que estos ofrecen desempeñando un papel de expertos en la opinión del consumidor. Hay algunos casos de bodegas que nos encontramos que tienen una oferta bien preparada y estructurada pero que les mancan intermediarios para llegar al consumidor. Es la diferencia entre la que llamamos puesta en valor y puesta en el mercado. Aquello que es la puesta en valor integra el patrimonio y el recurso que conjuntamente con la mano de obra pueden llegar a generar un atractivo pero que no completa un producto sin una puesta en el mercado para que haya un consumidor. Este consumidor es el que genera un producto porque es el que pide a través de un intermediario, ya sea una agencia, empresa de servicios o centrales de reserva, poder adquirirlo. Esta deficiencia de actores puede llegar a ser clave cuando se rompe la cadena del producto enoturístico hacia el receptor que impide muchas veces la recuperación del esfuerzo económico hecho en primera instancia.

Esa falta de intermediarios ha hecho recapacitar sobre la aplicación de un nuevo modelo de comunicación en el enoturismo que pasa de una comunicación lineal bodega-distribuidorcliente a una multidireccional. Esta voluntad de establecer un diálogo directo no sólo con el cliente final, sino con el resto de agentes es para establecer una red de información y facilitar el feedback. Esta posibilidad de diálogo es muy importante, tanto para la bodega, para saber dónde puede mejorar, como para el cliente final, que tiene un canal a través del cual puede expresarse, compartir y consultar información. Aquí es donde entran en juego las redes sociales.

\section{Redes sociales}

El concepto de red social es un término que asociamos a Facebook o Twitter, pero la definición es más amplia. La descripción más acurada de Red Social seria: un conjunto de personas o entidades unidas por algún interés común y con las que se puede interactuar. Así mismo, con la tecnología actual, el concepto red social adquiere una nueva dimensión. Ponce, en el Observatorio Tecnológico de 2012 las define de esta forma: "Aparece el término de redes sociales online, pudiendo definirlo como aquellas estructuras sociales compuestas por un grupo de personas que comparten un interés común, relación o actividad a través de Internet, donde tienen lugar los encuentros sociales y se muestran las preferencias de consumo y de 
información mediante la comunicación en tiempo real. Esta comunicación bidireccional que ofrecen las redes sociales es dónde el enoturismo busca entablar una comunicación con el consumidor final para crear sinergias positivas que ayuden a mejorar la experiencia de estos. Si bien uno de los principales problemas que se podría detectar en el enoturismo es la falta de intermediarios, el marketing relacional es una cruzada importante que tiene este tipo de turismo para llegar al consumidor objetivo. De las estrategias de marketing digital, nos centraremos en el uso de las redes sociales. Para Telles (2010) la gestión de las RRSS “es una parte integrante de una gestión de la reputación online, una estrategia para organizaciones y personas que se preocupan por su presencia online y que además de optimizar, aumentan el número de visitas, generan conversaciones e interacciones y aumentan la popularidad de los links.

\section{Redes sociales en el enoturismo}

La elección del tipo de redes sociales dónde debe estar la empresa forma parte del plan de Social Media. Una vez hemos visto que tipo de redes y para qué sirven, pasaremos a observar si las empresas que se dedican al enoturismo en España están presentes en las principales redes sociales. Compés y Castillo afirman que las redes sociales sobre las suponen una revolución en el sector del vino no solo en la forma de comunicarse sino de socializar, buscar información o recomendaciones y comprar un producto. Las redes contribuyen, en gran medida, a dotar de mayor autenticidad a las zonas vitivinícolas (alojamientos, restaurantes o bodegas) a través de una comunicación directa bidireccional entre la marca y el consumidor.

Resaltamos también otro estudio de la compañía de investigación de mercados Nielsen que afirma que alrededor del $10 \%$ de las bodegas españolas llevan más de 6 años en las redes sociales, muy cerca del $38 \%$ lleva más de tres años (accedieron entre 2008 y 2010), casi un $40 \%$ entraron entre 2011 y 2012 y sobre un $10 \%$ de las que quedan aún no lo han hecho, pero piensan hacerlo en el corto plazo, en total sumando casi un $90 \%$. Además, un $97 \%$ de las bodegas analizadas está en Facebook siendo la red por excelencia, y un $65 \%$ tiene cuenta en Twitter. También resulta significativo, por una parte, que el $15 \%$ de las empresas analizadas dedique más de 10 horas semanales a mantener sus contenidos en dichas redes, aunque, por otra, tan sólo la mitad de las compañías incluye dichas redes sociales en sus estrategias de marketing. De hecho, sólo un $44 \%$ de las bodegas analizadas utilizaban herramientas analíticas para evaluar el impacto de sus acciones en las redes sociales.

\section{Metodología}

El enfoque que usaremos en esta investigación será de carácter exploratorio para hacer una fotografía general de cómo está la situación actual en las redes sociales en la región vitivinícola del Priorat. Los estudios exploratorios son investigaciones con el objetivo de dar una visión aproximada de una determinada realidad que ha sido poco explorada y reconocida en la cual es difícil formular hipótesis precisas (Hakim, 1987).

\section{Resultados}

En primer lugar, hemos analizado si las empresas de las cuales hemos delimitado la investigación tenían perfiles en las redes sociales. Todas ellas, como citamos en la 
introducción, tienen una voluntad de crear sinergias entre los diferentes actores en el enoturismo que tiene la comarca del Priorat, así que la gran mayoría disponen de web dónde de una forma más simple o compleja exponen su negocio. Así pues, cabe destacar que dentro de la muestra tenemos establecimientos que ofrecen diversidad de servicios y dónde hallamos diferencias entre estos grupos que no son del todo homogéneos. De la muestra un $80 \%$ de las empresas poseen un perfil en Facebook siendo los alojamientos el grupo con la tasa más alta, un $81 \%$. La segunda red social con más perfiles entre las empresas dedicadas al enoturismo en el Priorat es Twitter con el 52\% siendo las bodegas el grupo con la tasa más alta, un $61 \%$. Finalmente encontramos Instagram en tercer lugar con dos puntos menos que Twitter, un 50\% y los alojamientos han sido el grupo con una ratio más alta de perfiles, $52 \%$.

Figura 1. Índice de Perfiles de Redes Sociales

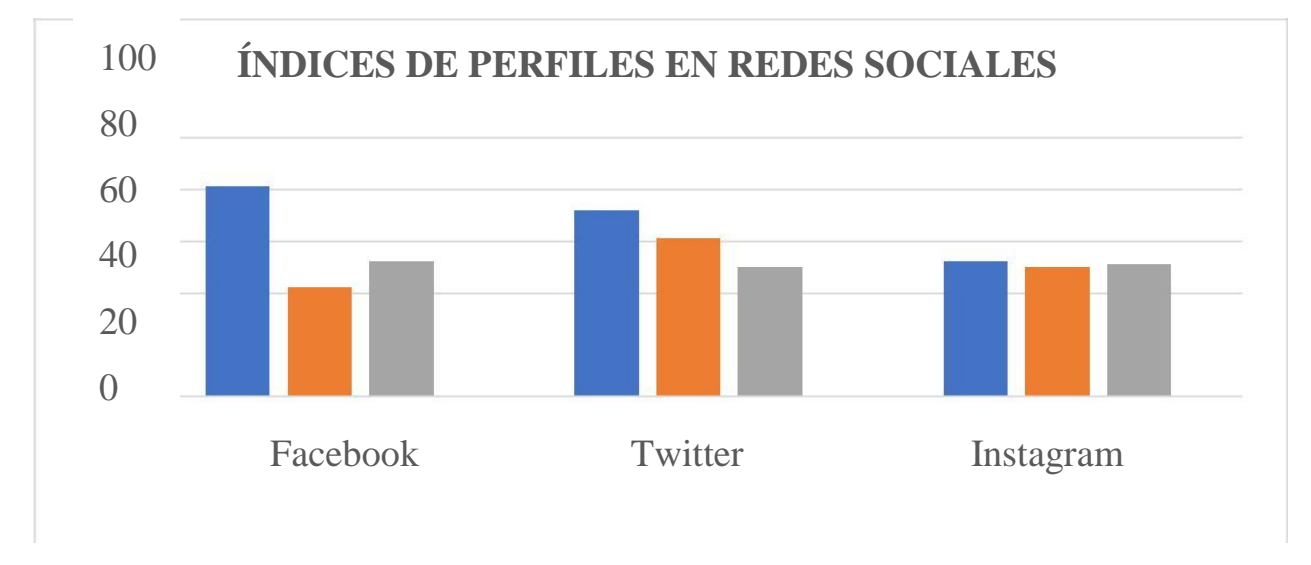

Fuente: Elaboración propia

Si comparamos las ratios obtenidos con los datos del Informe de IAB Spain publicado el 2015 sobre el uso de las redes sociales en las empresas enoturísticas, vemos que el uso de Facebook queda aún más lejos que el número de empresas que tienen perfil en Twitter.

Figura 2. Comparación Uso de Redes Sociales Privot vs T. España

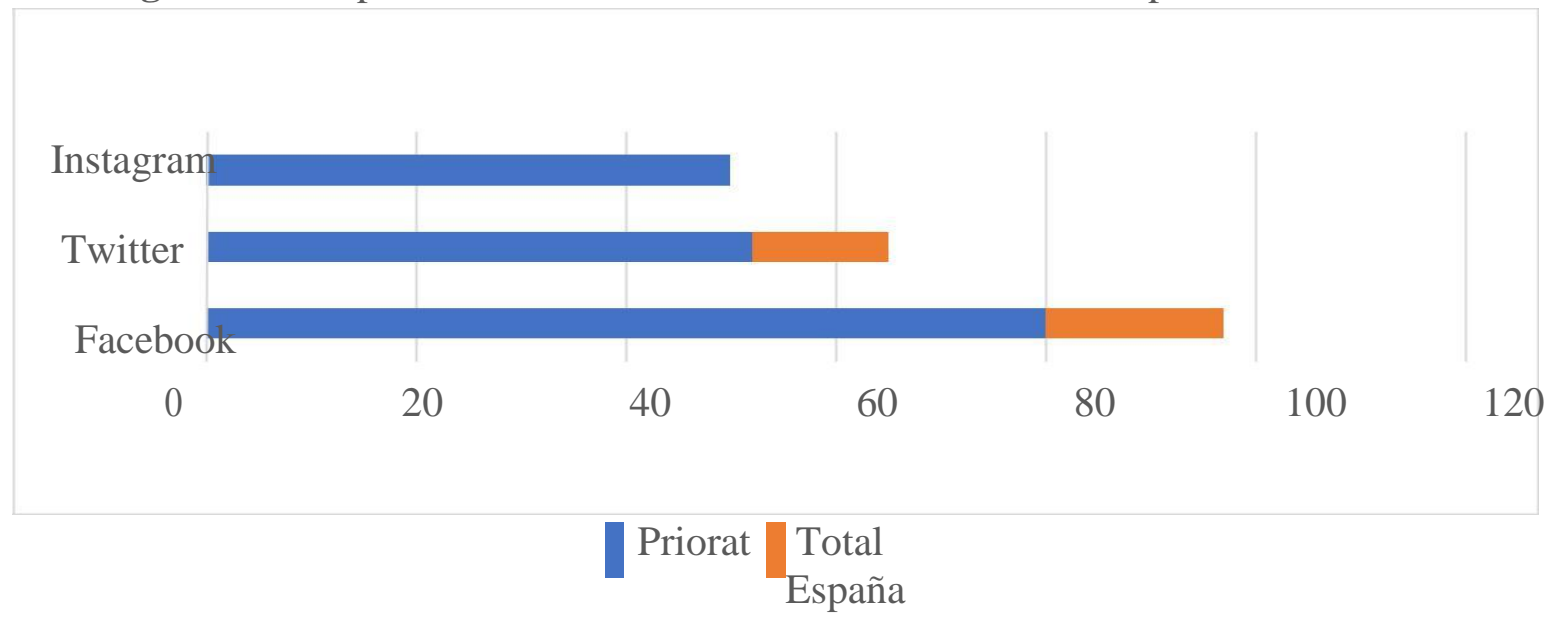

*No hay datos Instagram en España 
Finalmente, empresas que tengan perfiles en las tres redes encontramos un $31 \%$ de la muestra. Otras plataformas que encontramos con link directo a la web de la empresa han sido con orden de importancia, TripAdvisor (18\%), Linkedin (15\%) y Pinterest (12\%).

\section{Análisis Facebook}

Como hemos comentado anteriormente, Facebook es, des de lejos, la red social que cuenta con más presencia de las empresas enoturísticas del Priorat, con un $80 \%$. Antes de realizar una monitorización de las publicaciones durante el último año en el perfil Facebook de cada una de las empresas, desarrollaremos varias hipótesis que nos ayuden a explicar la interacción con los usuarios con el fin de tener un retorno a toda aquella actividad que se genere en las redes sociales.

H1: A mejor información en la página de Facebook y facilidad para contactar con la empresa, más seguidores en la página.

H2: A mayor actividad en las redes, (dialogo con otras páginas, captar la atención del usuario, número alto de posts per day) mayor será el nivel de engagement de la página.

En primer lugar, tenemos el índice About que nos calcula si se está dando información y contexto correctamente a todos los usuarios que accedan a la página. Entre los datos hay, números de contacto, localización, web y un poco de historia de la empresa. El último dato es el que suele faltar en las páginas analizadas. El promedio que encontramos es de 0,83, encontrando los índices más altos en los alojamientos siendo Clos Figueras y Hostal Sport las empresas que ostentan el máximo índice $(0,94)$. El índice promedio es un número alto por lo que se desprende que hay una clara voluntad de estar en las redes y no es un complemento al marketing tradicional.

Si segmentamos la muestra en tres grupos diferenciándolos por su índice About. Siendo el más bajo $n=0,59$ y el más alto $n=0,94$ la mediana nos lleva a situar el grupo 1 de 0,94 a 0,85 , el grupo 2 de 0,84 a 0,75 y el grupo 3 de 0,74 a 0,59. El porcentaje que obtenemos de promedio en cada grupo es el siguiente:

Figura 3. Porcentaje promedio de grupo

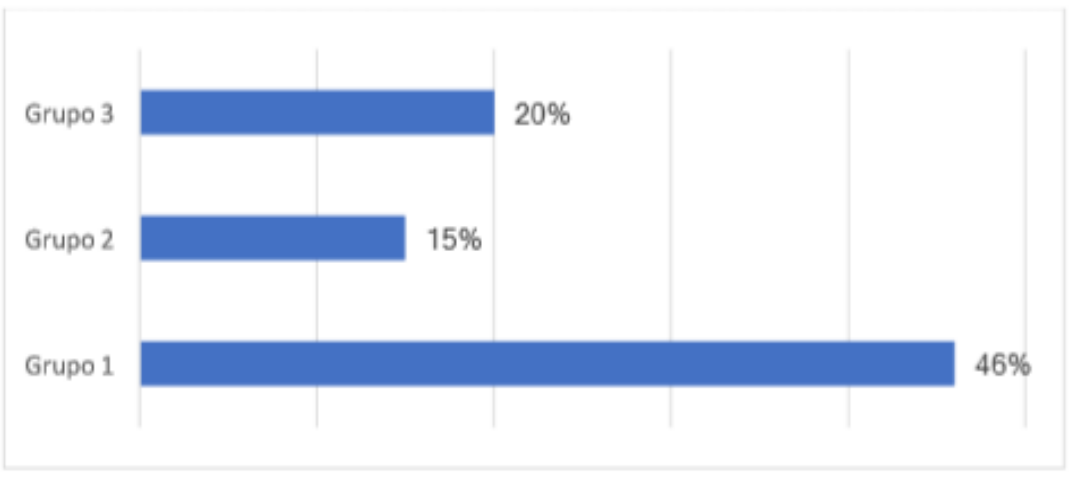

Fuente: Elaboración propia 
Así pues, se cumple la primera igualdad por la que el grupo con el perfil más completo es el que tiene una tasa más alta de engagement. Juntamente con el nivel de actividad medimos el número de post per day y la duración en las redes dependiendo del nivel de presencia que tenga la página web dentro de la red social. Aquí encontramos una mediana de 0,2 posts per Day siendo los más activos L'escolaneta de Baronia con 0,5 y Hostal Sport con 0,9. En este caso pasaremos a comprobar si este nivel de actividad coincide con un mayor engagement en las redes. Los volvemos a diferenciar en tres grupos siguiendo el nivel de actividad. Siendo el índice más alto $\mathrm{n}=0,86$ y el más bajo $\mathrm{n}=0,12$ hacemos una dispersión para encontrar tres grupos:

$$
\begin{aligned}
& \text { Grupo1 }(0,86-0,71) \text { à42\% } \\
& \text { Grupo } 2(0,71-0,36) \text { à37\% } \\
& \text { Grupo } 3(0,36-0,12) \text { à18\% }
\end{aligned}
$$

Se cumple la segunda hipótesis, ya que el grupo que genera más actividad en las redes es el que tiene mayor nivel de interacción. Seguimos con la tercera hipótesis. El número de fans nos indica si la información que publican en las páginas es suficiente atractiva para los usuarios de la red social. Este dato es útil con su medida a lo largo del tiempo para saber cuándo ha funcionado la actividad en la red para atraer nuevos usuarios. Al haber analizado si estamos presentes en las redes, nos falta monitorizar la actividad de los usuarios. Para analizar esta variable usaremos el índice de PTAT (People Talking about that). Este índice toma importancia en los medios sociales ya que muestra la influencia de manera conjunta de acciones como los me gusta, comentarios, compartido, etiquets, check-ins, etc.

Para calcularlo se relaciona con el número de fans de la página y te permite hacer un cálculo de lo activa y popular que es la página. Esta relación se establece con la variable engagement. Como comprobaremos a continuación, tener una amplia base de usuarios que han puesto "Me gusta" a la página web no garantiza un nivel de engagement alto por qué a más usuarios es más difícil que todos sean intensivos.

Figura 4. Nivel de Engagement

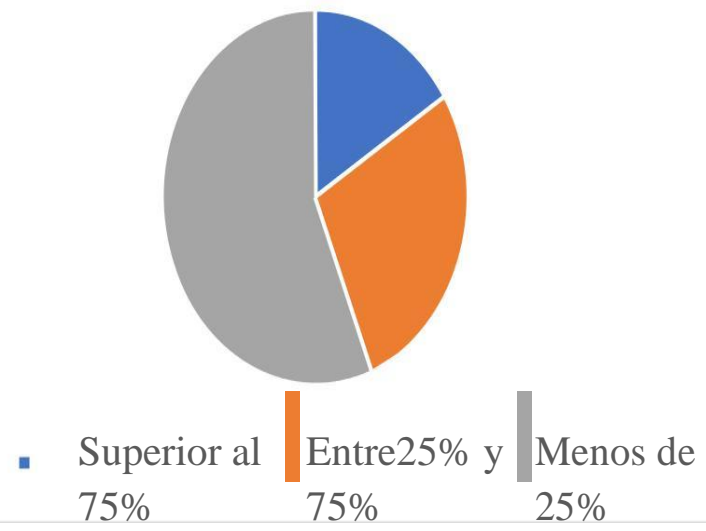

Fuente. Elaboración propia 
Cómo vemos en el gráfico de arriba, un $56 \%$ de las empresas tienen un nivel de engagement inferior al $25 \%$ por lo que nos indica que más de la mitad de las empresas no están llegando a hacer que sus seguidores expresen sus opiniones o preguntas a través de la red social. Al no hacer partícipes sus clientes hay un mayor riesgo de que la fidelidad de estos irá en descenso. Dentro del grupo de empresas con un nivel de engagement superior al 75\% la mediana está cerca de 1500 usuarios, mientras que el total es de 1831. Para hacerlo más claro dividiremos las empresas en tres grupos según el número de seguidores con una dispersión por lo que obtendremos:

\section{Grupo 1 (Menos de 750) à 22\%}

Grupo 2 (750-1500) à30\%

Grupo 3 (Más de 1500) à23\%

Por lo que podemos deducir que el nivel de engagement no se relaciona con tener un número excesivo de seguidores, sino que nos tendremos que concentrar en otros tipos de variables para explicar este alto índice. Cómo podemos observar, el número máximo de seguidores que permite tener un alto índice de interacción es 1500, cuando se pasa este número, el nivel de engagement baja. Una de las posibles respuestas es el nivel de calidad de información que aportamos y a quién va dirigida. El nivel de información en su página de Facebook y la inclusión de concursos y publicaciones para interactuar con otras páginas ayuda a generar más interacción. En un caso práctico vemos que El Brogit Guiatges realiza eventos y comparte información con otras páginas que ayuda a indexar y llegar a conectar con un target más amplio.

\section{Análisis Twitter}

De las empresas analizadas, un 52\% usan la red social Twitter. Las empresas que usan esta herramienta, un 95\% usan Facebook también, por lo que podríamos deducir que es una herramienta complementaria para generar tráfico web. La estrategia de las empresas en la comunicación se basa en llegar al máximo número de seguidores, pero muchos autores coinciden en señalar también que el éxito en la capacidad de difundir el mensaje está en la calidad de la interacción con los receptores. La herramienta TwitAtonomy nos permitirá conocer la audiencia que tiene cada una de las empresas analizadas y como se relacionan cada una de ellas a través de la plataforma social twitter. En este apartado intentaremos establecer una conexión entre el número de seguidores y la actividad de la empresa en la red con los tuits diarios con el nivel de engagement. En este caso el nivel de interacción lo mediríamos con el porcentaje de retuits sobre el nivel de tuits y el promedio de menciones de otros usuarios. En este caso probaremos con las siguientes hipótesis:

- H1: A mayor nivel de usuarios y actividad en las redes, mayor es el nivel de interacción y engagement con los usuarios.

- H2: Las empresas con una mayor base de usuarios tienen una estrategia clara y continuista para fomentar la interacción entre usuarios y la fidelización de clientes 
Para comprobar las hipótesis vamos a agrupar las empresas según su número de seguidores, así nos quedarían 3 grupos: menos de 500, de 500-1500 y más de 1500. Con esta segmentación podremos comprobar si la primera de las afirmaciones es verdadera.

Grupo1 (menos de 500 seguidores) à 0,26

Grupo 2 (entre 500 y 1500 seguidores) à 0,69

Grupo 3 (Más de 1500 seguidores) à 0,62

Con estos resultados vemos que un mayor nivel de usuarios no cumple un mayor nivel de interacción con los usuarios, sino que hay un nivel máximo a lo cual cada cuenta puede aspirar a generar más actividad, que como vemos en la imagen de abajo, es de 1500 usuarios. Una vez superado este número es difícil generar tanto tráfico a un nivel tan elevado de usuarios. A continuación, pasamos a verificar la segunda hipótesis que busca establecer una relación entre la actividad de la cuenta y el nivel de interacción con los usuarios. En este caso también realizaremos una dispersión para segmentar las empresas en tres grupos.

Grupo 1: Entre 0 y 0,2 posts diarios à0,34

Grupo 2: Entre 0,2y 0,4 posts diarios 0,68

Grupo 3: Más de 0,4 posts diarios 2,10

Como podemos observar más arriba, la hipótesis se confirma ya que el grupo con un mayor número de actividad son los que generan más interacción en las redes. Como podemos observar en la tabla de arriba, el grupo 3 formado por las empresas con una mediana de más de 0,4 posts diarios es el que genera más interacción entre los usuarios y con la propia empresa. Aunque aquí también entra en juego, en menor cantidad, la variable de la calidad del contenido generado. Un ejemplo de ello es Clos Figueras, con 1856 seguidores, que intenta fomentar la compartición y los links con otras instituciones dentro del enoturismo de la comarca del Priorat mediante etiquetas y menciones con el fin de llegar a un número de seguidores más amplio y obtiene un $23 \%$ de la tasa de engagement. Por otro lado, tenemos a el Celler de Capçanes que, aunque su actividad es medianamente alta (0,43 tuits diarios), la calidad de estos no es para nada comparable, con el ejemplo que hemos visto anteriormente. Esto genera poco interés a los usuarios y lo vemos reflejado es su baja tasa de mención por tuit, un 5\%.

Tabla 1. Resumen de perfiles

\begin{tabular}{lccc}
\hline \multicolumn{1}{c}{ Empresa } & Followers & Posts & Engagement\% \\
\hline Clos Galena & 1014 & 61 & 13,8 \\
Hotel Lotus Priorat & 1253 & 275 & 10,4 \\
Celler mas Roig & 1020 & 98 & 8,3 \\
Clos Figueres & 1051 & 94 & 6,9 \\
Cooperativa flaset marca & 1178 & 487 & 6,1 \\
l' escoleta apartmanet turistics & 1268 & 543 & 3 \\
\hline
\end{tabular}

Fuente: Elaboración propia 
Observamos que justamente los perfiles con más posts este último año son los que tienen el menor nivel de engagement por lo que confirmamos que la segunda hipótesis quedaría nula.

\section{Conclusiones}

Del listado de empresas analizadas la presencia en las redes sociales aún tiene potencial crecimiento. Si bien vimos que la mediana de las zonas más importantes de vino en España es de un 97\% de presencia en Facebook y un 67\% Twitter, las empresas del Priorat aún tienen camino por recorrer. En este punto también cabe destacar el auge de Instagram como el canal más dinámico de cara al futuro ya que el $80 \%$ del tráfico de internet ya proviene de Smartphones. Así pues, Instagram va a tener mucho más peso de cara al futuro para la comunicación de las empresas y las empresas enoturisticas se tienen que empezar a adaptar a las posibilidades que puede explotar esta plataforma social.

Existe una diferenciación entre el uso de las redes sociales entre bodegas y alojamientos. Si bien en bodegas se usa más Twitter, en alojamientos se está usando más la plataforma Facebook. Esto nos lleva a la conclusión que no se está realizando una planificación clara del uso de las redes sociales y se está desaprovechando el potencial que puede aportar cada red social que complementando su uso se puede llegar a hacer una oferta comunicativa potente.

Para dirigirte y captar a aquellos usuarios que aporten una mayor interacción es clave el nivel de actividad que genere cada empresa a través de las redes sociales. Es por esto que en el análisis intentamos conectar la cifra de contenido generado con el nivel de engagement. Si bien vemos que en Facebook y Twitter si se cumple, en Instagram no. La diferencia la vemos por qué en las dos primeras redes, las empresas están creando contenido de cualidad aprovechando el potencial del canal. Cuando comprobamos la información que suministran, detectamos que aquellas bodegas que refuerzan con información los atributos del producto que venden o los encantos del entorno provocan una mayor interacción con los usuarios. En cambio, en Instagram no se cumple la premisa de a mayor nivel de contenido mayor interacción con los usuarios, esto nos lleva a afirmar que aún falta un mayor conocimiento de esta red social pero que tiene aún mucho potencial por ofrecer.

La realización de todas estas mejoras tiene un hándicap importante en el Priorat, la falta de infraestructura que garantice una cobertura total y rápida de internet. Es por esto que la demanda de unas mejoras en las condiciones tecnológicas es imprescindible para el crecimiento de la zona vitivinícola. Con estas conclusiones llegamos a poder afirmar que las redes sociales están produciendo cambios en la forma como comunican las empresas hacia sus clientes. 


\section{Bibliografía}

Bello L y Cervantes M (2002) La propuesta de un modelo positivo del proceso de compra de vino Cuadernos Aragoneses de Economía

Caldevila, D (2010) Las redes sociales, tipología, uso y consumo de las redes 2.0 en la sociedad digital actual UCM

Calvo, D y Blázquez, F (2007) Análisis del valor proporcionado por la marca de origen en los vinos de calidad Universidad de A Coruña

Calvo, D. y Blázquez, F. (2004), Comercialización de vinos de calidad: marca de fabricante vs. marca de distribuidor, ITEA

Calvo, D. (2002). Estrategias de marca en vinos con denominación de origen: Un análisis desde la óptica de los directivos. Investigación y Marketing

Calvo, D y Blázquez, F (2007) Análisis del valor proporcionado por la marca de origen en los vinos de calidad Universidad de A Coruña

Compés y Castillo (2014) La economía del vino en España y el Mundo Publicaciones Cajamar

Hall, M (2000) Wine Tourism arround the world British Library

Hall, M, \& Mitchell, R. (2001) Wine and food tourism Special Interest Tourism: Context and Cases.

Lopez, G Rodriguez, G Viera, R (2013) Revisión de la literatura científica sobre enoturismo en España

Mora, P Castaing, P. (2006). Buenas prácticas en marketing del vino: 20 estudios de casos de vinos del mundo. Ed. Mundi Prensa, Madrid

Prat, J (2014) Las rutas del vino del empordà y del priorat y su dinámica relacional Estudios Geográficos

Serrano, D (2015) Aproximació territorial a l'enoturisme com a fenomen de desenvolupament local a Catalunya Tesi Doctoral UB

Tolosa,L (2017) Marketing del Enoturismo Tolosa Wine Books

Van der Weig, M (2009). Tendencias e Innovación en el mercado español del vino. Nuevos conceptos, nuevas ideas. Universitat Politècnica de València

Enotur: una nueva herramienta de información enoturistica (2011) Tendències Enotur Núm 1.

Llorens, A El Priorat no puede ser un destino 'low cost' de enoturismo Entrevista a Enric Calvo 4/1/2016 La Vanguardia

Instagram le come el terreno a Facebook 9/2/2018 Eldiario.es

Observatorio turístico de las rutas del vino de España (2010) Asociación Española de ciudades de vino

Modelos de distribución del vino en España (2015) Informe Nielsen 2015 Indicadores de desarrollo sostenible para destinos turísticos (2005) OMT 
Madrid El vivid genera prop de mig milió d'euros d'impacte econòmic a la costa brava 3/5/2016 Cupatges.cat

El 95\% dels enoturistes que han visitat Cataluña tenen la intenció de tornar-hi 5/4/2016 Cupatges.cat

El gasto y la estancia media de los enoturistas vuelve a augmentar (2017) ACEVIN Informe sobre la demanda del turismo

Navarro, N Catalunya no sap explicar al món que som un gran país de vins Entrevista a Lluis Tolosa 10/11/2017 Revista Via Empresa

Propuesta de plan enoturistico de Cataluña (2016) Agencia Catalana de Turismo

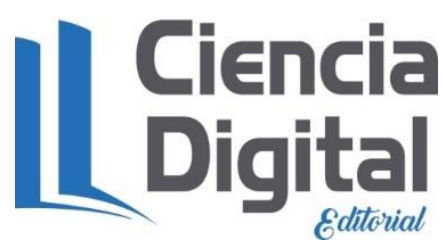




\section{PARA CITAR EL ARTÍCULO INDEXADO.}

Quero Guerrero, J., Caicedo Aldaz, J., Cedeño Alcivar, L., \& Zúñiga Chamorro, C. (2019). Impacto de las redes sociales en el sector del enoturismo del Priorat. Visionario Digital, 3(3), 84-99. https://doi.org/10.33262/visionariodigital.v3i3.647

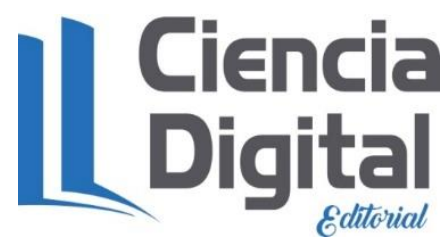

El artículo que se publica es de exclusiva responsabilidad de los autores y no necesariamente reflejan el pensamiento de la Revista Visionario Digital.

El artículo queda en propiedad de la revista y, por tanto, su publicación parcial y/o total en otro medio tiene que ser autorizado por el director de la Revista Visionario Digital.
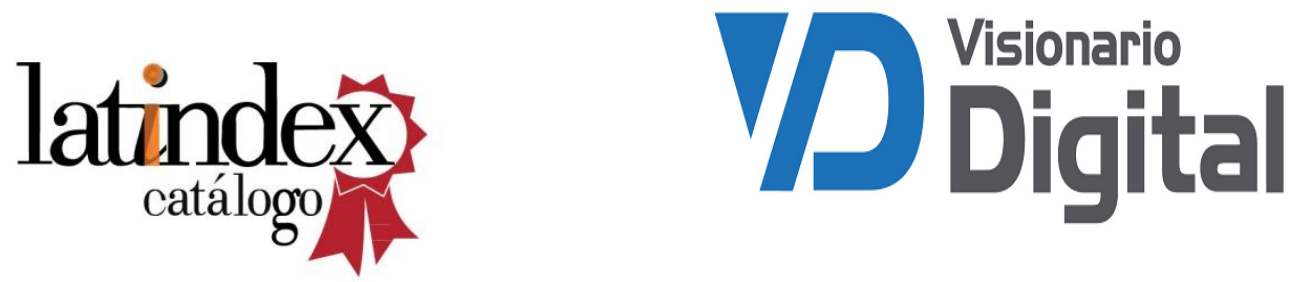